\title{
Zonal concentration of some geophysical process intensity caused by tides and variations in the Earth's rotation velocity
}

\author{
B. Levin ${ }^{1}$, A. Domanski ${ }^{1}$, and E. Sasorova ${ }^{2}$ \\ ${ }^{1}$ Institute of Marine Geology and Geophysics, Far East Branch of RAS, Yuzhno-Sakhalinsk, Russia \\ ${ }^{2}$ Shirshov Institute of Oceanology of Russian Academy of Sciences, Moscow, Russia \\ Correspondence to: B. Levin (levinbw@mail.ru) \\ Received: 9 February 2013 - Revised: 16 October 2013 - Accepted: 28 October 2013 - Published: 6 January 2014
}

\begin{abstract}
We analyzed what kind of fundamental physical phenomena can be responsible for the generation of the anomalous latitudinal zones of the seismic activity, and the hotspots, and some other geophysical processes. The assessment of tidal effect contribution to the earthquake preparation process is discussed. A disk model of the Earth's rotation was proposed. The model is acceptable for the homogeneous Earth and for the heterogeneous one. The disk model explains the nucleation of two maximums of the gradient of the moment of inertia over latitude with respect to the Equator. Effects of the variations in the Earth's rotation angular velocity were estimated and the possible features caused by the rotation velocity instability were described. The variations in the relative velocity of the Earth's rotation (dimensionless value $v \approx(T-P) / P$ ) are approximately equal upon the average to $10^{-8}$, where $T$ is the observed length of day for the Earth, and $P$ is the astronomical day. These variations lead to the occurrence of the additional energy estimated as $10^{20} \mathrm{~J}$.

The authors proposed the hypothesis of a pulsating geoid based on effects of the Earth's rotation features, and tidal forces, and conception of critical latitudes in the solid Earth. This hypothesis may highlight the phenomenon of zonal intensification of some geological processes in the solid Earth (the seismic activity, and hotspot location, and major ore deposit locations).
\end{abstract}

\section{Introduction}

A zonal amplification of the seismic activity at the middle latitudes was already denoted in the last century (Gutenberg and Richter, 1949; Mogi, 1979; Sun, 1992). It was revealed
(Levin and Sasorova, 2009a; Sasorova et al., 2013) that seismic activity of the planet is almost absent at the poles and at the polar caps, and has clearly expressed two peaks in the middle latitudes of the Northern Hemisphere and the Southern Hemisphere $\left(40-50^{\circ} \mathrm{N}\right.$ and $\left.20-35^{\circ} \mathrm{S}\right)$, and has the stable local minimum near the Equator $\left(10-20^{\circ} \mathrm{N}\right)$. Similar distributions are obtained as for a density of released energy (Fig. 1a), and for a density of the seismic events (Fig. 1b). Because of the fact that most earthquakes are concentrated along the boundaries of lithospheric plates, normalizing of earthquake number and released energy by the length of the lithospheric plate boundaries in every single latitudinal belt was used (Levin and Sasorova, 2009b). Such normalizing determines the average number of earthquakes generated per every $100 \mathrm{~km}$ of plate boundary. The worldwide seismic catalog (the International Seismological Centre catalog (ISC, UK)) was used. The data processing of 250000 seismic events with $M \geq 4$ from 1964 to date was carried out.

The stability of obtained bimodal distributions in time, in space, and for different latitudinal scales $\left(2,5\right.$, and $\left.10^{\circ}\right)$ was proved (Sasorova et al., 2013). It was also shown that the bimodal structure of the empirical distributions remains for shallow events, and for intermediate and deep earthquakes.

Thus the attempts to explain the revealed effect by the tidal forces were undertaken in works (Sun, 1992; Levin and Pavlov, 2003; Riguzzi et al., 2010).

Below we also discuss some problems connected with the influence of the tidal forces on the Earth's seismicity and on other geophysical processes. The direct relationship between the EQ origination and the tidal forces is not adequately validated now. However, it should be noted that the total influence of the tidal forces may have a distinct nonlinear effect. 

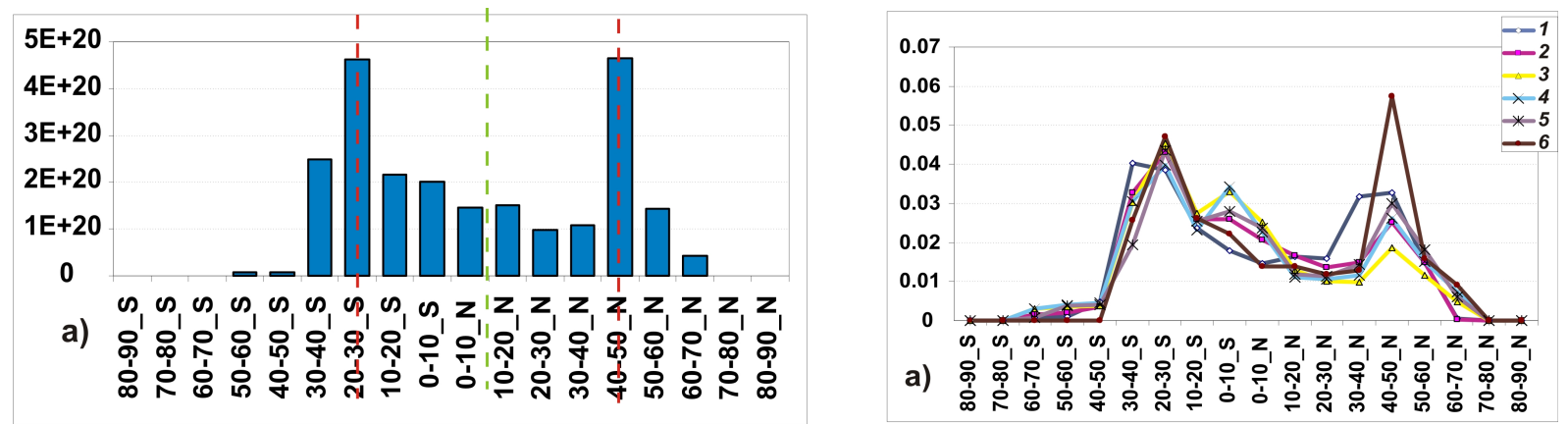

Fig. 1. The distribution of the energy density over latitude (a); the latitudinal distributions of the relative seismic event density for 6 magnitude ranges (b): 1 for $4.0 \leq M<4.5,2$ for $4.5 \leq M<5.0,3$ for $5.0 \leq M<5.5,4$ for $5.5 \leq M<6.0,5$ for $6.0 \leq M<6.5,6$ for $6.5 \leq M$. The data were adopted from the (ISC UK) catalog.

We also analyzed the latitudinal distributions of the major ore deposits in the Earth using the GIS system based on the "Largest Mineral Deposits of the World (GIS, 2006)". The version used contains data about 1242 major and supermajor ore deposits. It was revealed that the latitudinal distributions of deposit number and deposit density (in $10^{\circ}$ latitude belts) have a bimodal character: the peaks at $20-30^{\circ} \mathrm{S}$ and $40-60^{\circ} \mathrm{N}$, and the local minimum at $10-20^{\circ} \mathrm{N}$ and almost zero values at the high latitudes (Sasorova et al., 2013).

Thus the objective of this work is the search for the model combination that could consolidate the simple mechanical analysis of body rotation processes and the elements of the pulsating geoid model, taking in account the concept of critical latitudes and consequences of the planet rotation instability.

\section{The tidal forces contribution in the seismic process}

According to the tide theory (Melchior, 1983), the maximum of the tidal energy is observed in the Southern Hemisphere and the Northern Hemisphere at latitude $45^{\circ}$, and the zero values are marked at the poles and at the Equator.

The same results were obtained in the theoretical work (Levin and Pavlov, 2003) dedicated to the assessment of the energy accumulated in the Earth's lithosphere due to the effects of the external and the internal forces. According to this model, the total sum of all forces affected on every element of the Earth's lithosphere in the state of equilibrium has to be equal to zero. The largest part of the energy in the lithosphere is generated by the gravity force and the centrifugal one. Its interaction determines the shape of the planet and compression ratio of the Earth (ellipticity). The additional free energy is released under the influence of the external forces, and an essential part of this energy is due to the tidal forces. A similar opinion was expressed in the work (Sun, 1992), where maximums of the tide-latitude dependence were first compared with peaks of the seismicity latitude distribution.
The structure of the Earth crust rock where earthquakes are generated should be considered as a heterophase system consisting of the solid rock skeleton and the channels occupied by fluid-gas composition, which is a chemically active medium. The tide periodical impact on the Earth geospheres leads to the fluid movements along the channels, to alternating load, and to the alternate activation of the fluid that has an adsorption property. The fluid interaction with the rock massive brings to the strength degradation of the rock in accordance with the Rehbinder effect (Levin et al., 2010). Longterm exposure of the fluid as a surface-active material to rupture walls leads to a sharp reduction in the rock strength of $10-100$ times.

Analysis of the tide influence on the seismic process fulfilled in the work (Morgounov et al., 2006) showed that the trigger effect of the tidal periodic components on earthquake occurrence depends on the period of the tidal component. It was revealed that tidal component SSa (182.62 days), month component MM (27.55 days), and semi-month component MSF (14.77 days) manifested the statistical significance of the connection between the tidal phases and the earthquake occurrence. However, the short-term tidal harmonics (O1, $\mathrm{P} 1, \mathrm{~K} 1, \mathrm{~N} 2)$ with the diurnal and the semi-diurnal periods did not detect a similar connection.

The short-period tidal forces are especially powerful. However, the influences of weak but long-period tidal forces (monthly, semiannual and annual) are more effective than the influence of several times more powerful short-period tidal forces (Sun, 1992; Morgunov et al., 2006). A correlation between the EQ occurrence and short-period tidal forces is very low, while it becomes a very considerable value for the longperiod tidal forces.

The chemical interaction of the ascending fluid on the solid skeleton in the case of the long-period tides continues on a significantly longer time interval than for the shortperiod tide. Thus the duration of the effect is more important than its magnitude.

The long-period tides cause the slow alternating deformations in the rocks, promote the fluid motion from the depths 
to the surface, help the development of the micro-cracks in the rocks under the impact of the Rehbinder effect, and lead to the rupture accumulation and the creation of the conditions for the seismic fault nucleation (Kasahara et al., 2003).

Thus the long-period tidal effects in accordance with the modern ideas influenced the seismic processes more effectively. Evidently, along with the direct action of the tidal forces, it is necessary to take into account the tidal friction effects and the mechanism of dissipation in the heterogenic medium, and the viscosity and the thermal conduction effects in real rocks (Scholz, 1998).

Today, it is obvious that the tidal force influence on the Earth's lithosphere manifests itself in quite complicated ways, and there is no direct reaction of tidal effects to the process of the seismic event generation. However, the long-term repeated summarized impact of the tidal forces with the various periods could lead to a very sufficient nonlinear effect.

\section{A disk model of the Earth and variations in the moment of inertia}

A model describing the development of two symmetric zones of the instability in solid Earth with respect to the Equator is proposed. Thus the attempt to search the relationship between the rotation process characteristics of the Earth and the features of the observation data listed above was carried out.

The rotating body is regarded as the ellipsoid of revolution consisting of the sum of the infinitely thin disks perpendicular to the rotation axes (Fig. 2a). We shall consider that the ellipsoid of revolution is an approximation of the geoid. A moment of inertia of a rotating body and the derivative of the moment of inertia with respect to the distance (polar radius) were calculated. In this case the moment of inertia of the body is equal to the sum of moments of inertia of all disks (Levin et al., 2011). The disk model does not take into consideration the instability of the Earth's rotation velocity and any processes varied in time.

The moment of inertia of a rotating infinite thin circular $\operatorname{disk}(j(z))$ with radius $r$ is equal to:

$j(z)=\frac{\pi}{2} r^{4} \sigma=\frac{\pi}{2} R^{4}\left(1-\frac{z^{2}}{H^{2}}\right)^{2} \sigma$,

where $R$ and $H$ are the equatorial and the polar radius of the Earth, respectively, and $\sigma$ is the surface density.

The moment of inertia of a layer of the revolution ellipsoid with layer thickness $h$ is equal to:

$$
\begin{aligned}
J(h) & =\frac{\pi}{2} \rho R^{4} \int_{0}^{h}\left(1-\frac{z^{2}}{H^{2}}\right)^{2} \\
\mathrm{~d} z & =\frac{\pi}{2} \rho R^{4} H\left(t-\frac{2}{3} t^{3}+\frac{1}{5} t^{5}\right),
\end{aligned}
$$
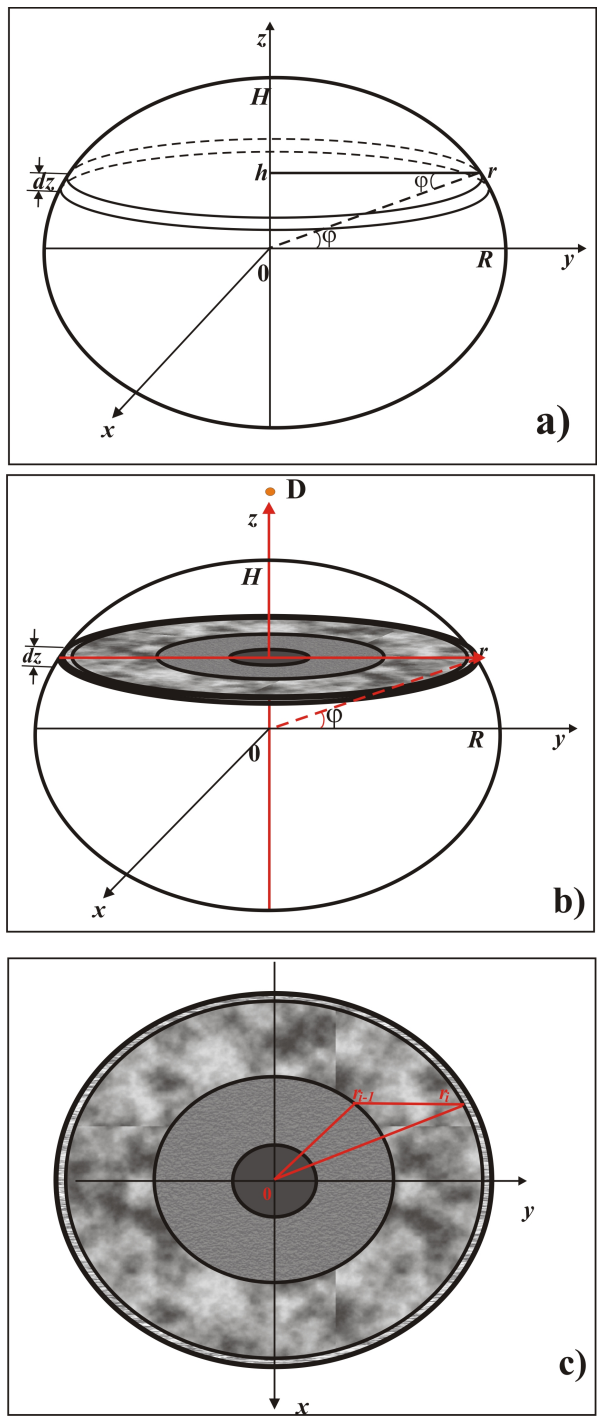

Fig. 2. The sketch of the HomED model (a), the sketch of the HetED model (b), and the vertical view on the disk plane shown on (b) from point $\mathrm{D}$ (c). Here $R$ is the equatorial Earth radius and $H$ is the polar radius, $\varphi$ is the latitude of a given infinitely thin disk, $h$ is the distance between the Earth's center and the disk plane, and $r_{i-1}$ and $r_{i}$ are outer radiuses of the two consecutive rings. The displayed rings do not identify with any determined geospheres of the Earth. This sketch is only an illustration of the calculation method of the moment of inertia for every ring.

where $t=h / H$, and $\rho$ is body density. The $\rho$ value for the homogeneous Earth disk model is equal to $5.52 \mathrm{~g} \mathrm{~cm}^{-3}$.

Then the inflection point of the moment of inertia as the function of the polar radius $(J(h))$ is determined by the expression $H\left(t-2 / 3 t^{3}+1 / 5 t^{5}\right)$ in Eq. (2) - and its location is defined by $h_{*}=H / H \sqrt{3}$. This point corresponds to latitude $35^{\circ} 15^{\prime} 22^{\prime \prime}$ for the homogeneous Earth.

The calculations were performed both for the homogeneous in density Earth disk model (HomED) and for the 
heterogeneous one (HetED). In the last case the Earth consists of several tens of the concentric geospheres (ellipsoids) with the same value of the compression. The infinitely thin disk that is parallel to the equatorial plane crosses the Earth at the given latitude, and it consists of several tens of the coaxial rings (Fig. 2b, c). In the case of the HetED model all geospheres situated at a given latitude are included in the calculations. To calculate the moment of inertia of infinitely thin disks perpendicular to the rotation axes for the HetED model, it is necessary to define the following parameters of each ring: the average density of the medium $\left(\rho_{i}\right)$, and the outer radius of the ring $\left(r_{i}\right)$, where $1 \leq i \leq N$ and $N$ is the total number of ellipsoids. Every ring has the constant density inside the ring $\left(\rho_{i}\right)$, and its volume is calculated as the difference between the volumes of the two consecutive rings (Fig. 2b). The total moment of inertia of the disk composed of several rings is defined as the sum of the moment of inertia of all rings comprising the given disk.

Thereto we used three world-famous models of the internal structure of the Earth: the Bullen-Haddon (1967) model, the PREM model (Anderson, 2007), and the AK135-F model (Montagner and Kennett, 1995). Each of the mentioned models of the internal structure of the Earth consists of several tens of geospheres. Every model determines the number of concentric geospheres $\left(n_{i}\right.$, which are equal to 49,89 , and 145 , respectively), the average value of density for each ellipsoid layer $\left(\rho_{i}\right)$, and its outer radius. The boundary of the mantle outer core and the boundary of the inner core and the outer core are described in these tree models by several ellipsoids taking into account the complicated processes occurring on these boundaries.

The sketch of the HetED model is shown in Fig. $2 b$ and c. The last one is the vertical view on the disk plane shown in Fig. $2 b$ from point $D$. It should be noted that the simplest sketch of the Earth is manifested. The displayed rings do not identify with any determined geospheres of the Earth. This sketch is necessary only for illustration of the calculation method of the moment of inertia of every ring.

The critical latitudes are located at $\pm 35^{\circ}$ for the homogeneous Earth, and these latitudes are located at latitudes $26^{\circ} 18^{\prime} 36^{\prime \prime}, 26^{\circ} 20^{\prime} 16^{\prime \prime}$, and $26^{\circ} 17^{\prime} 24^{\prime \prime}$, respectively, for the heterogeneous Earth. The three calculated values practically coincide $\left(26^{\circ} 18^{\prime} \pm 1^{\prime}\right)$. It should be noted that the number of ellipsoids in the three models mentioned above varies from 49 (Bullen-Haddon model) to 145 (AK135-F), but the differences in "the critical latitude" locations are no more than $\pm 1^{\prime}$. The liquid outer core and the solid inner core are considered in the analyzed disk models as the parts of the infinitely thin disks with corresponding density. In this manuscript we presented the disk model only at qualitative level. All details of the calculations were discussed in the work (Levin et al., 2011).

The gradient of the moment of inertia of the infinitely thin disks $(G(h))$ as a bimodal function becomes the maximum values at two symmetrical points with respect to the Equator

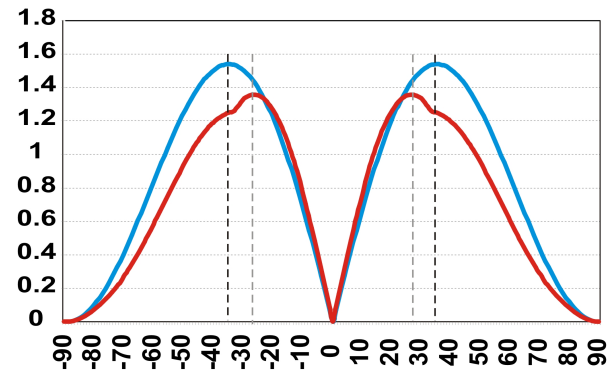

Fig. 3. The latitudinal distributions of a dimensionless gradient of the moment of inertia of the infinitely thin disks (the $G(h)^{*}$ values) are presented for the homogeneous Earth (blue line) and for the heterogeneous Earth (red line). Horizontal axis is angular latitudes. Vertical axis gives the $G(h) *$ values. Maximum values marked as dashed lines.

determined as critical geocentric latitude. The dimensionless gradient $G(h)^{*}$ was determined as the $G(h)$ value normalized by the averaged value $G(h)$ for the homogeneous Earth ( $h$ varied as $0<h<H$ ). The coefficient of normalization is equal to $2 \pi \rho R^{4} / H$. The latitudinal distributions of the dimensionless gradients $\left(G(h)^{*}\right)$ for the homogeneous Earth and for the heterogeneous Earth are presented in Fig. 3.

It was shown that the existence of "the critical latitudes" and their location do not depend on the Earth's compression value $-\varepsilon$ (in the case of the insignificant ellipticity) - and depend only on geometric properties of the rotating body.

The common tendency of the activation of some physical processes at the middle latitudes for certain rotating celestial bodies (the Earth, the Sun, the Moon, Jupiter, etc.) should be noted now. For example, the well-known phenomenon is that a period of revolution of the Sun layers depends on heliocentric latitude. These periods vary from 25 days for the layers near the Sun's Equator to 29 days for the layers located near the Sun's polar caps. The peaks of the gradient of the revolution period are located at latitudes $\pm 40^{\circ}$, and just approximately at these latitudes arise the sunspots. The critical latitudes may also be observed as a feature of other rotating celestial bodies.

\section{The Earth's figure and critical latitudes}

The Earth's figure had been shaped under the influence of gravity and centrifuge potentials as a spheroid (geoid) with an ellipticity:

$\varepsilon=(R-H) / R$,

where $R$ and $H$ are the equatorial radius and the polar radius of the planet, respectively. The spheroid radius vector is determined by the general expression:

$r=R_{0}\left(1-(2 / 3) \varepsilon P_{2}(\sin \phi)\right)$, 


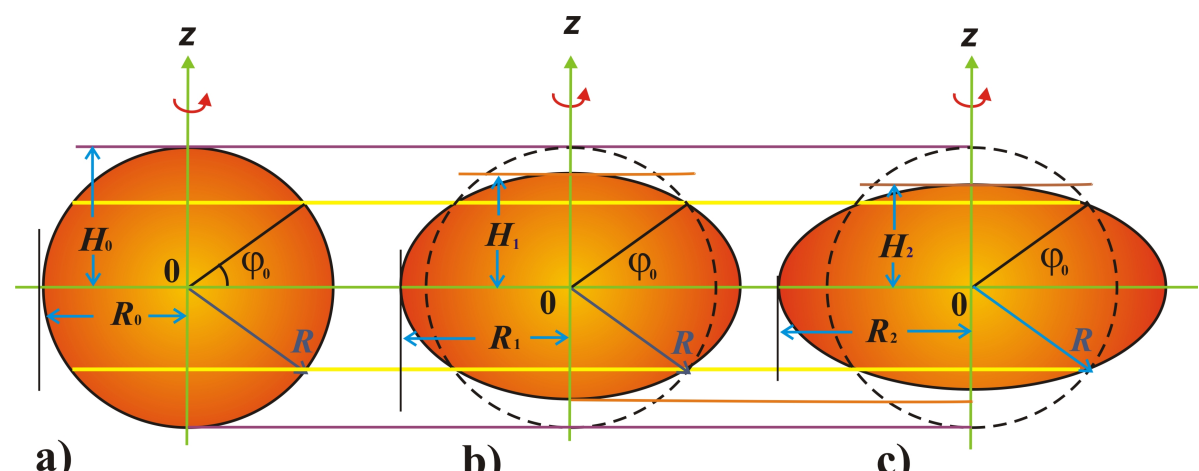

a)

b)

c)

Fig. 4. The sketch of the rotating celestial body: three fragments for three different values of angular velocity. The angular velocity value $\omega_{0} \approx 0$ (a); the angular velocity is $\omega_{1}>\omega_{0}(\mathbf{b})$; the angular velocity is $\omega_{2}>\omega_{1}>\omega_{0}$ (c). The yellow solid line denotes critical latitude for three fragments of the rotating body. The dotted black circles on the (b) and (c) fragments indicate the spheres whose volume is equal to the volumes of rotating ellipsoids.

where $R_{0}$ is the radius of the sphere, whose volume is equal to the volume of the spheroid, $\varphi$ is the geocentric latitude, and $P_{2}(\sin \phi)=1.5 \cdot \sin ^{2} \phi-0.5-$ a zonal spherical function of the second order.

It is evident from Eq. (3) that the radius vector $r=R_{0}$ coincides with the average radius of the planet at critical latitudes $\left(\phi_{0}= \pm \arcsin (1 / \sqrt{3})= \pm 35^{\circ} 15^{\prime} 52^{\prime \prime}\right)$ and does not depend on its ellipticity.

The scheme of the spheroid evolution and the critical latitude appearance is presented in Fig. 4. It contains three fragments with three celestial bodies with different rotation angular velocities: $\omega_{0}, \omega_{1}, \omega_{2}$. Each fragment is described by the set of the parameters: the rotation angular velocity $\left(\omega_{i}\right)$, the equatorial radius $\left(R_{i}\right)$, the polar radius $\left(H_{i}\right)$, the compression ratio of the body $\varepsilon_{i}$ (or ellipticity) and the inertia moment $\left(I_{i}\right)$. The fragment (a) presents the rotating body with the following parameters: $\omega_{0} \approx 0, \varepsilon_{0} \approx 0, R_{0} \approx H_{0}, I_{0}$. The parameters of the rotating body on the fragment (b) become values: $\omega_{1}>\omega_{0}, \varepsilon_{1}>\varepsilon_{0}, R_{1}>R_{0}, H_{1}<H_{0}, I_{1}>I_{0}$. The dotted black circles on the fragments (b) indicate a sphere whose volume is equal to the volumes of rotating ellipsoids, and the centers of these bodies are located at the same point. The intersection plains of two such bodies are located at latitudes $\pm \varphi_{0}$. The fragment (c) presents the rotating body with parameters: $\omega_{2}>\omega_{1}, \varepsilon_{2}>\varepsilon_{1}, R_{2}>R_{1}, H_{2}<H_{1}$, and $I_{2}>I_{1}$, but the intersection plains in this case are also located at latitude $\pm \varphi_{0}$. The locations of the intersection plains of two bodies do not vary with the changing of the angular rotating velocity; these locations remain constant at the latitudes $\pm \varphi_{0}$ called "critical latitudes".

On the basis of equations for the geopotential at the Equator (Stacey and Davis, 2008), at the Pole, and at the critical latitude, we revealed the theoretical formula for the ellipticity of a planet that does not contain the body moment of inertia $\varepsilon=\frac{R_{0}^{3} \omega^{2}}{\gamma M}$, where $\gamma$ is the gravitation constant, and $M$ is the Earth's mass.

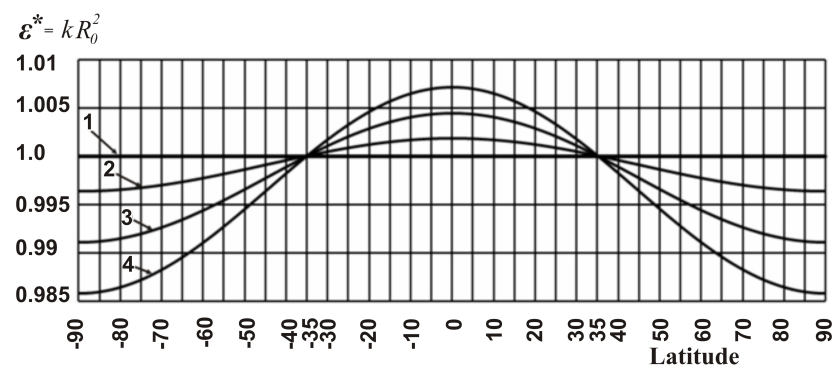

Fig. 5. The dependence of the Gaussian curvature normalized to the sphere curvature $\varepsilon^{*}=\kappa R_{0}^{2}$ over latitude for different ellipticity values: 1 for $\varepsilon_{0}=0-$ (sphere), 2 for $\varepsilon=0.00135,3$ for $\varepsilon=0.00335$ - (the Earth), 4 for $\varepsilon=0.00535$. The vertical axis is a normalized curvature.

The total (or Gaussian) curvature of the rotation ellipsoid surface is determined, as it is well known by the following equations:

$$
\begin{gathered}
\kappa=\frac{(1-\varepsilon)^{8 / 3}}{R_{0}^{2}} Q^{2}\left(\varepsilon, \sin ^{2} \phi\right), \\
Q=\frac{\sin ^{2} \phi+(1-\varepsilon)^{2} \cos ^{2} \phi}{\sin ^{2} \phi+(1-\varepsilon)^{4} \cos ^{2} \phi} .
\end{gathered}
$$

The dependences of the spheroid total curvature on the latitudes for different ellipticities $\varepsilon_{i}$ are given in Fig. 5. The total curvature values are normalized to the sphere curvature $\varepsilon_{0}$. The normalized curvatures are calculated as $\varepsilon^{*}=\kappa R_{0}^{2}$. One can see that common points of the intersection for the family of Gaussian curves with small ellipticity are situated at the critical latitudes. Using the series expansion for the total curvature, we can receive as its first approximation $\kappa=\kappa_{1}\left(1-(8 / 3) \varepsilon P_{2}(\sin \phi)\right)$, where $\kappa_{1}=1 / R_{0}^{2}$ is the total curvature of a sphere. Evidently, for the first approximation a total curvature of a spheroid at the critical latitudes does not 


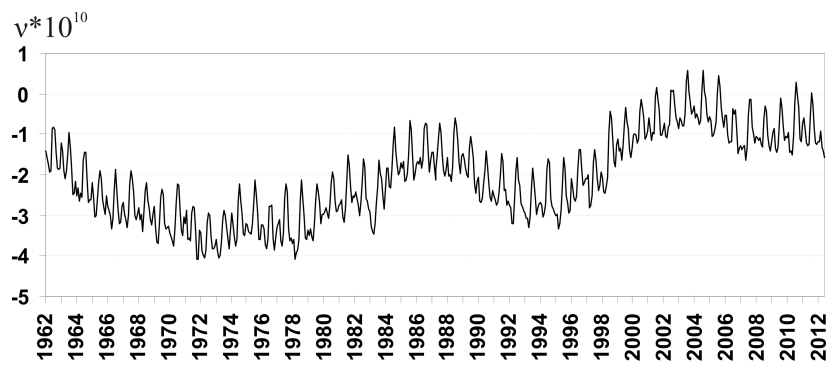

Fig. 6. The time series of the monthly averaged values of relative angular velocity of the Earth's rotation ( $v$ ) during the last $50 \mathrm{yr}$, where $v \approx(T-P) / P$; here $T$ is the Earth length of day (s), and $P$ is the astronomical day $-86400 \mathrm{~s}$. The vertical axis gives $v \times 10^{10}$.

depend on an ellipticity in the case where the sphere volume and the spheroid volume are equal.

It should be noted that the areas with the largest variation in the curvature values (maximum of the curvature gradient) are located near the critical latitudes.

\section{The variation in the Earth's rotation velocity}

The Earth's rotation energy is determined in the first approximation as the energy of the perfectly rigid body (sphere) with the constant moment of inertia and the constant angular velocity of the rotation. The average value of the Earth's rotation energy around the axis of revolution is approximately equal to $E=3 \times 10^{29} \mathrm{~J}$.

The change in the ellipticity of the rotating ellipsoid (geoid) involves a change in the total energy of the rotating body. Outer influences, and particularly a tidal friction of the Moon, and also interior effects, originated in a global mantle discontinuity, and gravity, and a chemistry differentiation of the Earth's interior, and other phenomena lead to a loss in the Earth's rotation velocity.

The variations in the rotation velocity value have been collected by survey by the IERS (IERS, 2000) since 1962. However, the data from 1656 are available now (McCarthy and Babcock, 1986).

The plot of the variation in the Earth's rotation velocity from 1962 to 2012 is presented in Fig. 6. The value of the relative variation in velocity is equal to $v \approx(T-P) / P$. Here $T$ is the Earth length of day (s), and $P$ is the astronomical day $-86400 \mathrm{~s}$ (the vertical axis in Fig. 6 gives $v \times 10^{10}$ ).

The results of the spectral analysis of the $v$ value are presented in Fig. 7. It should be noted that the relative variations in rotation velocity (per year) have an amplitude of about $10^{-8}$. Below it will be used for an assessment of additional energy value occurring due to the variations in the rotation velocity.

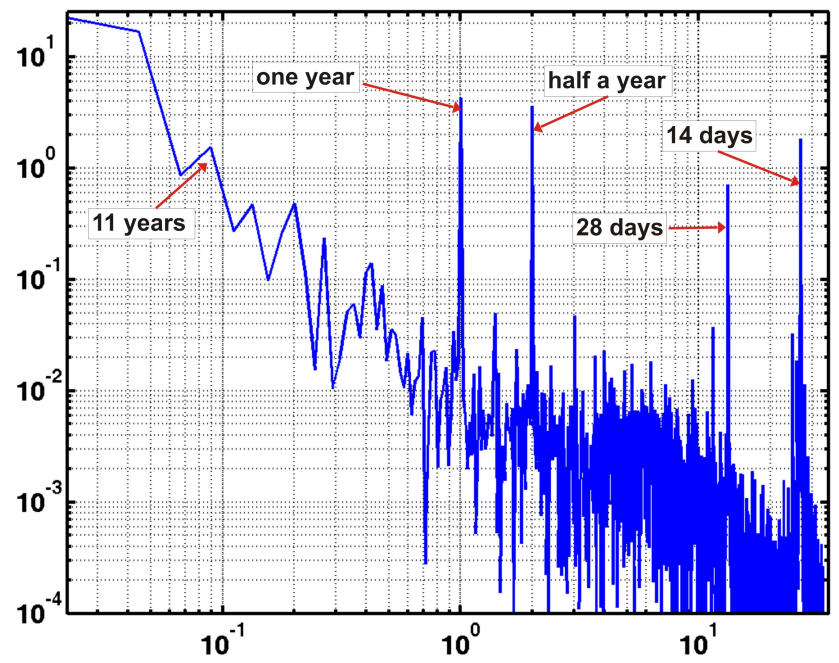

Fig. 7. Spectrum of monthly averages of the $v$ values on a log-log scale. The red lines with arrows indicate the representative peaks of the spectrum. The horizontal axis gives a number of cycles per year.

\section{The assessment of the Earth rotation excess energy}

The kinetic energy of the revolving body is described by the following expressions:

$E=1 / 2 \times I \omega^{2}, \quad$ where $I=\frac{2}{5} \times \frac{M R_{0}^{2}}{(1-\varepsilon)^{2 / 3}}$.

Let us designate:

$E_{1}=\frac{1}{5} M R_{0}^{2} \omega^{2}$, thus $E=\frac{E_{1}}{(1-\varepsilon)^{2 / 3}}$.

An expansion into a series of the expression (Eq. 6) gives:

$E=E_{1}+\frac{2}{3} \varepsilon E_{1}+O\left(\varepsilon^{2}\right)$,

where $O\left(\varepsilon^{2}\right)$ is the remainder term. Therefore the relative energy of a spheroid may be described as:

$E^{*}=\frac{E-E_{1}}{E_{1}}=\frac{2}{3} \varepsilon>0$,

and the relative radius vector of the spheroid becomes, according to expression (Eq. 3), equal to:

$r^{*}=\frac{r-R_{0}}{R_{0}}=-\frac{2}{3} \varepsilon P_{2}(\sin \phi)$.

This formula gives the deflection degree of the spheroid radius vector in comparison with the radius of the sphere. Thus kinetic energy will depend only on the ellipticity of a planet.

We will determine the dependence of the Earth's rotation energy on the variations in the angular velocity rotation. The derivative of energy of the rotating body is equal to:

$d E=2 E d \omega / \omega+1 / 2 \omega^{2} d I$. 
Thus, the total rotation energy had to increase at least to the value of $d E=2 E d \omega / \omega$ through the influence of the velocity variations, and the addition energy can be calculated as:

$d E=2 E^{*} d \omega / \omega=6 \times 10^{29} \times 10^{-8} \mathrm{~J}$

and may reach $10^{20}-10^{21} \mathrm{~J}$.

The analysis of the peculiarity of the Earth's rotation angular velocity performed on the basis of Newton's works by Maclauren, Clairaut, Poincare, and Veronnet (Poincare, 1902; Veronnet, 1912) allowed us to propose the model of the pulsating geoid. The growth in the spheroid rotation angular velocity leads to the increase in the equator radius, to the rise in the ellipticity and the kinetic energy of the rotating body. The changes in the angular velocity had to lead to pulsations of the spheroid with fixed location of the critical latitudes. The growth of the angular velocity also produced an extension of the medium in the Equator area and the compression in the polar zones. The decrease in the angular velocity leads to the opposite effect - the compression in the plane of the Equator and the extension along the polar axis. Such pulsations of the spheroid had to transfer the additional energy to the Earth's shell in comparison with the energy of the rotating of the perfectly rigid body.

\section{Conclusions}

The disk model of the Earth takes into consideration (regards) only the gradient of inertia of a rotating celestial body consisting of the sum of infinitely thin disks perpendicular to rotation axes. It was also manifest that just the ellipsoid figure properties could explain the existence of the "critical latitudes" on the basis of the disk model, but this model does not regard the instability of the velocity of the Earth's rotation.

An analysis of the effects of the zonal intensification of some observed geophysical processes on the Earth combined with the analysis of the ellipsoid figure properties allowed the authors to make sense of the complicated problem and to propose the hypothesis of pulsations of the geoid. The disturbed motion of the Earth in the Earth-Moon system together with other outer and inner influences leads to the instability of the planet's rotation angular velocity. The change in the angular velocity leads to the accelerations and decelerations of the geoid rotation that finally results in the growth and decline of the ellipticity alternately, i.e., to the pulsations of the geoid. The energy of these pulsations can be estimated by values near $10^{20} \mathrm{~J}$. It is comparable with the energy released annually from the earthquakes. So, the effect of the geoid pulsation may explain the phenomenon of the earthquake triggering and the intensification of the seismic process in the middle latitude region.

The maximum of the energy released because of the irregularity of the Earth's angular rotation velocity is located in the areas near the critical latitudes, and the zone of the intensifications of some geophysical processes are also connected with the critical latitudes.

Acknowledgements. This work was supported in part by the Russian Foundation for Basic Research (project no. 10-05-00116a and no. 13-05-00060a). We thank N. S. Sidorenkov for many helpful discussions.

Edited by: K. Tokeshi

Reviewed by: S. Na and another anonymous referee

\section{References}

Anderson, D. L.: New Theory of the Earth, 2nd Edn., Cambridge University Press, p. 400, 2007.

Bullen, K. E. and Haddon, R. A. W.: Derivation of an earth model from free oscillation data, P. Natl. Acad. Sci. USA, 58, 846-852, 1967.

GIS: Largest Mineral Deposits of the World, Commission on Geological Map of the World, CD-ROM, State Geological Museum, Moscow, 2006.

Gutenberg, B. and Richter, C. F.: Seismicity of the Earth and associated phenomena, Princeton University Press, Princeton, New Jersy, p. 284, 1949.

IERS: Annual Report, International Earth Rotation Service, Observatoire de Paris, Paris, 2000.

Kasahara, J., Toriumi, M., and Kawamura, K. (Eds.): The Role of Water in Earthquake Generation, University of Tokyo Press, Tokyo, p. 392, 2003.

Levin, B. W. and Pavlov, V. P.: The Influence of Astronomical Factors on Energy Density Variations in the Solid Shell of the Earth, Izvest. Phys. Solid Earth, 39, 601-606, 2003.

Levin, B. W. and Sasorova, E. V.: Bimodal Character of Latitudinal Earthquake Distributions in the Pacific Region as a Manifestation of Global Seismicity, Dokl. Earth Sci., 424, 175-179, doi:10.1134/S1028334X09010383, 2009a.

Levin, B. W. and Sasorova, E. V.: Latitudinal distribution of earthquakes in the Andes and its peculiarity, Adv. Geosci., 22, 139145, doi:10.5194/adgeo-22-139-2009, 2009b.

Levin, B. W., Rodkin, M. B., and Sasorova, E. V.: Specific Features of the Seismic Regime in the Lithosphere: Manifestations of the Deep Aqueous Fluid Action, Izvest. Phys. Solid Earth, 46, 452460, 2010.

Levin, B. W., Sasorova, E. V., and Domanski, A. V.: Hydrodynamic Instability at Mid-Latitudes of the Earth Caused by the Rotation of the Planet, Dokl. Earth Sci., 438, 641-644, doi:10.1134/S1028334X11050059, 2011.

McCarthy, D. D. and Babcock, A. K.: The length of day since 1656, Phys. Earth Planet. Inter., 44, 281-292, 1986

Melchior, P.: Tides of the planet Earth, Pergamon Press, Oxford, 1983.

Mogi, K.: Global variation of seismic activity, Tectonophysics, 57, 43-50, 1979.

Montagner, J. P. and Kennett, B. L. N.: How to reconcile body-wave and normal-mode reference Earth models?, Geophys. J. Int., 125, 229-248, 1995.

Morgounov, V. A., Boyarsky, E. A., and Stepanov, M. V.: Tidal wave period and seismicity, Dokl. Earth Sci., 406, 112-115, 2006. 
Poincare, H.: Figures d'equilibre d'une masse fluide, C. Naud, Paris, 1902.

Riguzzi, F., Panza, G., Varga, P., and Doglioni, C.: Can Earth's rotation and tidal despinning drive plate tectonics?, Tectonophysics, 484, 60-73, 2010.

Sasorova, E. V., Levin, B. W., and Rodkin, M. V.: A common feature in latitudinal dependence of different geophysical processes occurring on the rotating Earth, Adv. Geosci., 35, 15-21, doi:10.5194/adgeo-35-15-2013, 2013.
Scholz, C. H.: Earthquakes and friction laws, Nature, 391, 37-42, 1998.

Stacey, F. D. and Davis, P. M.: Physics of the Earth, Cambridge University Press, New York, 2008.

Sun, W.: Seismic energy distribution in latitude and a possible tidal stress, Phys. Earth Planet. Inter., 71, 205-216, 1992.

Veronnet, A.: Rotation de l'ellipsoïde hétérogène et figure exacte de la Terre, Journal de Mathématiques Pures et Appliqués, 6, 331463, 1912. 